\title{
ТАКТИКА ПРИ ОСТРЫХ ВОСПАЛИТЕЛЬНЫХ ОСЛОЖНЕНИЯХ ДИВЕРТИКУЛЯРНОЙ БОЛЕЗНИ
}

\section{Коротких Кирилл Николаевич}

Студент, Лечебного факультета Федеральное Государственное бюджетное образовательное учреждение высшего образования Воронежский государственный медицинский университет имени Н.Н. Бурденко Министерства здравоохранения Российской Федерации

Аннотация: Изучены результаты лечения 52 пациентов с диагнозом дивертикулярная болезнь ободочной кишки. Из них у 41 пациента ограничились консервативной терапий, а 11 больным были выполнены различные виды хирургических вмешательств. Женщин 27(65,9\%), мужчин 14 $(34,1 \%)$. Средний койко-день составил $8,7 \pm 0,5$ дней. Средний возраст в группе хирургического лечения составил 51,4 $\pm 3,9$ лет, женщин 5 (45,5 \%), мужчин 6 (54,5\%). Средний койко-день составил $15,2 \pm 1,8$ дней.

Тактика лечения зависела от размеров абсцесса, выявленного при УЗИ или РКТ.

Ключевые слова: Дивертикулярная болезнь, абсцесс брюшной полости, перитонит, консервативная терапия, хирургическая тактика.

\section{TACTICS FOR ACUTE INFLAMMATORY COMPLICATIONS OF DIVERTICULAR DISEASE}

\section{Korotkikh Kirill Nikolaevich}

\begin{abstract}
The results of treatment of 52 patients diagnosed with diverticular colon disease were studied. Of these, 41 patients were limited to conservative therapy, and 11 patients underwent various types of surgical interventions. 27 women (65.9\%), 14 men (34.1\%). The average bed-day was $8.7 \pm 0.5$ days. The average age in the surgical treatment group was $51.4 \pm 3.9$ years, 5 women $(45.5 \%), 6$ men (54.5\%). The average bed-day was $15.2 \pm 1.8$ days.

Treatment tactics depended on the size of the abscess detected by ultrasound or RCT.
\end{abstract}


Keywords: Diverticular disease, abdominal abscess, peritonitis, conservative therapy, surgical tactics.

Заболеваемость дивертикулезом и дивертикулярной болезнью толстой кишки увеличивается во всем мире. Несмотря на то, что большинство пациентов остаются бессимптомными на протяжении долгой жизни, распространенность дивертикулярной болезни толстой кишки, включая острый дивертикулит становится значительным бременем для системы здравоохранения с точки зрения прямых и косвенных затрат. В настоящее время основное внимание уделяется определению правильного терапевтического подхода путем тестирования различных методов лечения. К сожалению, надежные доказательства эффективности медицинской стратегии для предотвращения рецидива острого дивертикулита все еще не выработаны.

Дивертикулез - это наличие множественных дивертикулов полого органа. При дивертикулезе ободочной кишки дивертикулы по происхождению приобретённые, по строению - ложные, по морфофункциональным особенностям - пульсионные. Преимущественная локализация дивертикулов ободочная кишка, в прямой кишке дивертикулы образуются крайне редко. Дивертикулярная болезнь (ДБ) - это заболевание, клинические, морфологические и функциональные проявления которого определяются патологическими изменениями как минимум одного из дивертикулов В основе развития дивертикулярной болезни, в отличие от дивертикулеза, лежат воспалительные изменения в стенке дивертикулов.

С чем же связано возникновение дивертикулов? В первую очередь - это изменение классических свойств соединительной ткани. А повышение внутрибрюшного давления приводит к пролапсу слизистой через слабые участки кишечной стенки [1].

Задержка эвакуации содержимого из тела дивертикула через его узкую шейку приводит к образованию плотного комка, называемого фекалитом. При полной обструкции шейки дивертикула в его теле развиваются процессы воспаления, а в просвете накапливается экссудат. Если в результате размягчения фекалита воспалительным экссудатом не происходит его эвакуация через шейку в просвет кишки, то развивается реактивное воспаление окружающих дивертикул тканей, а затем - пропитывание окружающих тканей воспалительным экссудатом. Дефекты стенки при этом заполняются грануляционной тканью, которая находится в постоянном контакте с агрессивным содержимым толстой кишки с высокой концентрацией 370 
микроорганизмов. И содержимое кишки контактирует не со стенкой кишки, а околокишечной клетчаткой, создавая условия для перехода процесса в хроническую форму и развития рецидивов дивертикулита [2].

Выделяют два типа дивертикулеза - «западный» и «восточный». При «западном» типе дивертикулеза, в первую очередь, поражаются левые отделы ободочной кишки. Однако нередким бывает сегментарное поражение ободочной кишки дивертикулами, например, они определяются в сигмовидной и поперечной ободочной кишке, а в других отделах их нет. Преимущественная географическая распространённость «западного» типа дивертикулеза - США, Канада, Европа, Россия, Австралия. «Восточный» тип дивертикулеза характерен для стран Дальнего Востока и Юго-Восточной Азии. При этом дивертикулы локализуются в слепой и восходящей кишке. Распространённость его в десятки и сотни раз ниже, чем его «западный» вариант. Так же частота дивертикулеза увеличивается с возрастом. Так, до 40 лет это заболевание диагностируется менее чем у 5\% населения, в возрасте 40-50 лет - у 5-10\%, 50-60 лет — у 14\%, у лиц старше 60 лет — в 30\%, старше 80 лет — в 60-65\% случаев. Среди мужчин и женщин распространенность заболевания приблизительно одинаковая, однако также есть небольшая зависимость от возраста - до 40 лет чаще болеют мужчины, а после 70 лет - женщины [3].

Вероятность перехода дивертикулеза в состояние дивертикулярной болезни составляет 5-20\%. При этом у 75\% больных развивается острый дивертикулит, а у $25 \%$ - другие осложнения. Перфорация дивертикула является 4-й по частоте среди причин экстренного хирургического вмешательства после острого аппендицита, перфоративной гастродуоденальной язвы и кишечной непроходимости, а также 3-й - при формировании кишечных стом. При дивертикулярной болезни частота развития внутрибрюшного абсцесса или перитонита составляет 3,5-4 случая на 100 тысяч человек в год $[4]$.

Универсальной классификации дивертикулярной болезни ободочной кишки не существует. Для применения в клинической практике, на наш взгляд, следует придерживаться классификации, разработанной Ассоциацией колопроктологов России (2014) [5]. Согласно этой классификации, наличие хотя бы одного дивертикула в толстой кишке позволяет установить диагноз ДБ.

Дивертикулярная болезнь принципиально разделяется на бессимптомную форму, неосложнённую форму с клиническими проявлениями и осложнённую форму. Бессимптомную форму констатируют, если в ободочной кишке имеется хотя бы один дивертикул и отсутствуют какие-либо клинические проявления 
заболевания. Неосложненная форма ДБ с клиническими проявлениями преимущественно проявляется функциональными нарушениями и болями при отсутствии каких-либо признаков воспалительных изменений в дивертикулах. Осложненная форма ДБ имеет место при развитии воспалительных реакций или толстокишечных кровотечениях. В осложненной форме ДБ выделяют острые и хронические осложнения [6] (табл. 1).

Таблица 1.

Классификация осложнений ДБ

\begin{tabular}{|c|c|}
\hline Острые осложнения & Хронические осложнения \\
\hline I. Острый дивертикулит & $\begin{array}{l}\text { I. I. Хронический дивертикулит } \\
\text { 1. рецидивирующее течение } \\
\text { 2. непрерывное течение } \\
\text { 3. латентное течение }\end{array}$ \\
\hline II. $\quad$ II. Острый паракишечный инфильтрат & II. $\quad$ II. Стеноз \\
\hline $\begin{array}{l}\text { III. Перфоративный дивертикулит } \\
\text { 1. периколическая флегмона } \\
\text { 2. абсцесс } \\
3 \text { гнойный перитонит } \\
\text { 4. каловый перитонит }\end{array}$ & $\begin{array}{l}\text { III. Хронический паракишечный инфильтрат } \\
\text { 1. рецидивирующее течение } \\
\text { 2. непрерывное течение }\end{array}$ \\
\hline \multirow[t]{2}{*}{ IV.Толстокишечное кровотечение } & $\begin{array}{l}\text { IV.Свищи ободочной кишки } \\
\text { внутренние } \\
\text { наружные }\end{array}$ \\
\hline & $\begin{array}{l}\text { V.Рецидивирующее толстокишечное } \\
\text { кровотечение }\end{array}$ \\
\hline
\end{tabular}

В мировой практике для определения распространенности воспалительного процесса при острых осложнениях широко применяется классификация Hinchey E.J. (1978) (табл. 2) [7,8]. На сегодняшний день тактика лечения определяется согласно этой модифицированной классификации.

Таблица 2.

\section{Классификация Hinchey E.J.}

\begin{tabular}{|c|l|}
\hline Классификация Hinchey E.J. (1978) & \multicolumn{1}{|c|}{$\begin{array}{c}\text { Модифицированная классификация E.J. } \\
\text { Hinchey (1984) }\end{array}$} \\
\hline I - Периколический абсцесс или инфильтрат & $\begin{array}{l}\text { I a - Ограниченное периколическое } \\
\text { воспаление (инфильтрат) }\end{array}$ \\
\hline & I b - Ограниченный периколический абсцесс \\
\hline
\end{tabular}


Продолжение Таблицы 2.

II - Тазовый, внутрибрюшной или ретроперитонеальный абсцесс
II - Тазовый, отдаленный внутрибрюшной или ретроперитонеальный абсцесс

III - Генерализованный гнойный перитонит

III - Генерализованный гнойный перитонит

IV - Генерализованный «каловый» перитонит

IV - «Каловый» перитонит

При 1 и 2 стадиях Hinchey тактика лечения зависит от размеров диагностированных абсцессов. Абсцессы развиваются в среднем у 15-20\% пациентов. У 52-74\% больных чрескожное дренирование абсцессов позволяет избежать экстренного оперативного вмешательства и выполнить в отсроченном порядке одноэтапную резекцию сигмовидной кишки. При диаметре абсцесса менее 3-4 см проводится парентеральная антибиотикотерапия [9,10,11,12].

В случае увеличения размеров абсцесса выполняется чрескожное дренирование абсцесса. При размерах абсцесса более 4 см или при наличии тазового абсцесса (2 стадия по Hinchey) пациентам выполняют дренирование под контролем компьютерного томографа или ультразвука. Чрескожное дренирование абсцессов является не только эффективным методом лечения, но и позволяет избежать экстренного хирургического вмешательства и выполнить радикальную операцию после полноценной подготовки, что уменьшает риск развития послеоперационных септических осложнений [13].

Пациентам с 3 и 4 стадиями осложнений острого дивертикулита показано экстренное оперативное лечение [14,15].

С января 2016 по октябрь 2021г года в колопроктологическом отделении БУЗ ВО ВОКБ №1 было пролечено 52 пациента с диагнозом дивертикулярная болезнь ободочной кишки. Из них у 41 пациента ограничились консервативной терапий, а 11 больным были выполнены различные виды хирургических вмешательств. Средний возраст в группе консервативной терапии составил 58,2 $\pm 1,8$ лет, женщин 27(65,9\%), мужчин 14 (34,1\%). Средний койко-день составил $8,7 \pm 0,5$ дней (наибольший 18 , наименьший 4). Средний возраст в группе хирургического лечения составил 51,4 $\pm 3,9$ лет, женщин 5 (45,5 \%), мужчин 6 (54,5\%). Средний койко-день составил 15,2 $\pm 1,8$ дней (наибольший 27, наименьший 8).

Консервативная группа. Значение C-реактивного белка при поступлении в группе консервативной терапии колебалось от 3,2 до 277 (среднее значение 
$30,4 \pm 7,5)$, после проведенного курса консервативной терапии показатель нормализовался - среднее значение $5,2 \pm 0,6$ (от 20 до 2) (рис. 1). Лейкоцитоз при поступлении составлял в среднем $10,2 \pm 0,4$, после курса терапии $6,4 \pm 0,2$ (рис. 2).

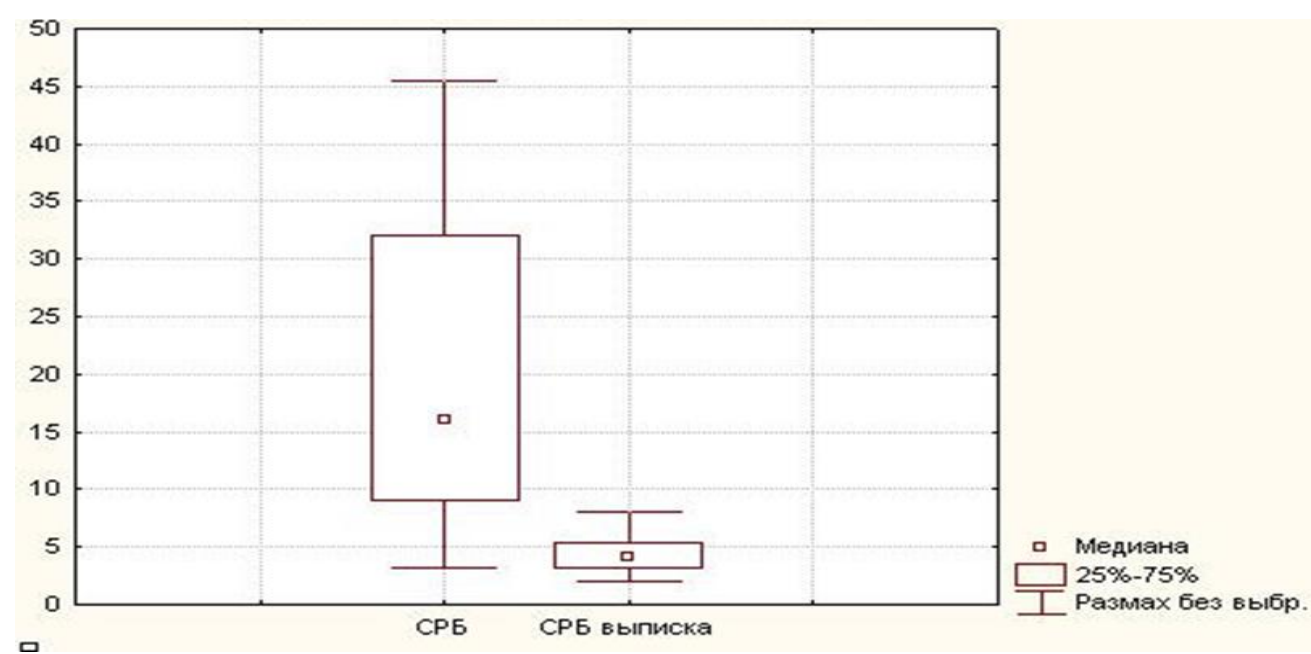

Рис. 1. Динамика СРБ в группе консервативной терапии

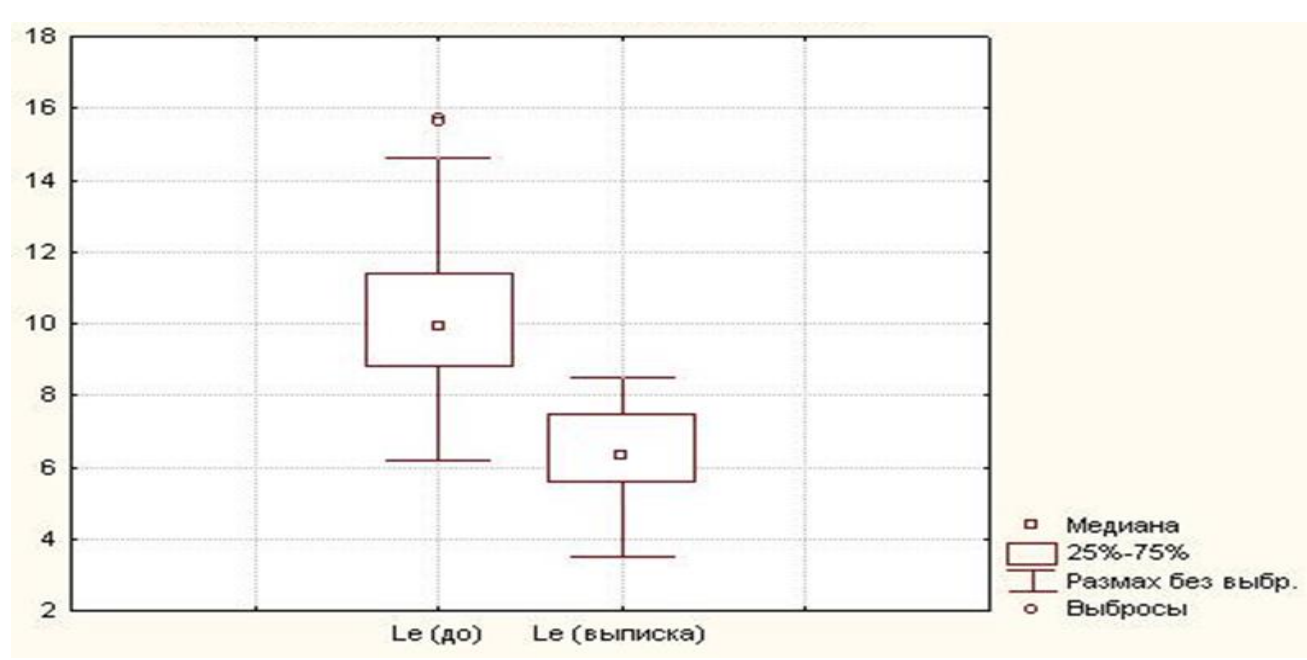

Рис. 2. Динамика лейкоцитоза в консервативной группе

Группа хирургического лечения.

Значение С-реактивного белка при поступлении колебалось от 25 до 318 (среднее значение $126,2 \pm 25,3$ ), после проведенного курса показатель нормализовался - среднее значение $6,7 \pm 0,6$ (от 10,6 до 4,5) (рис. 3). Лейкоцитоз при поступлении составлял в среднем $12,1 \pm 1,7$, после хирургического вмешательства $8,1 \pm 0,7$, а при выписке $5,9 \pm 0,6$ (рис. 4). 


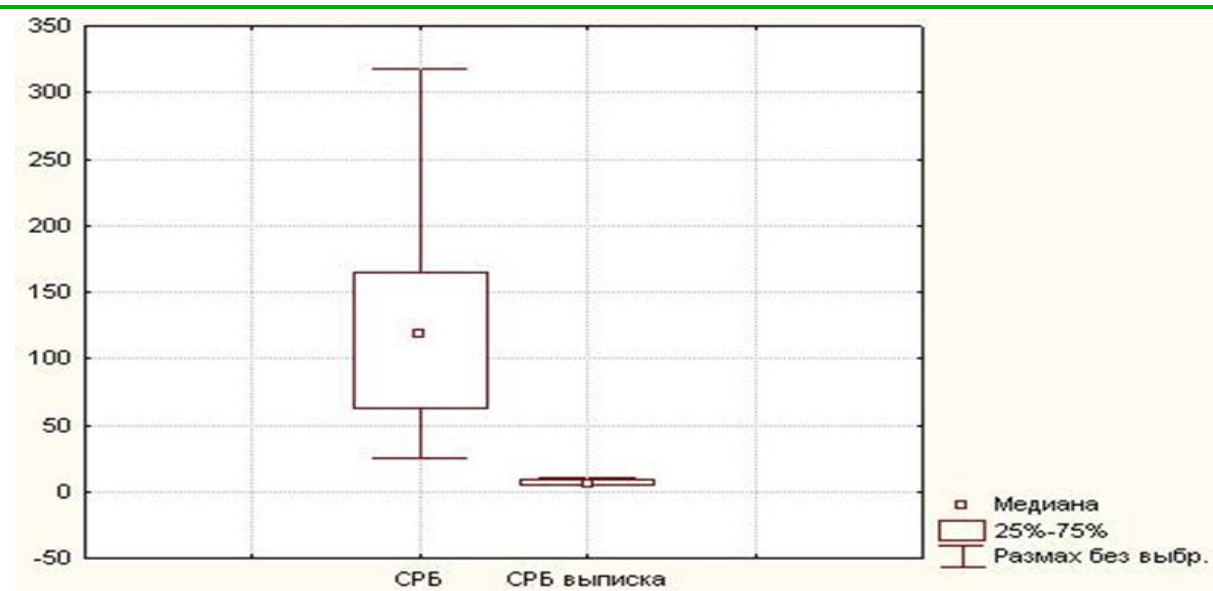

\section{Рис. 3. Динамика СРБ в группе хирургического лечения}

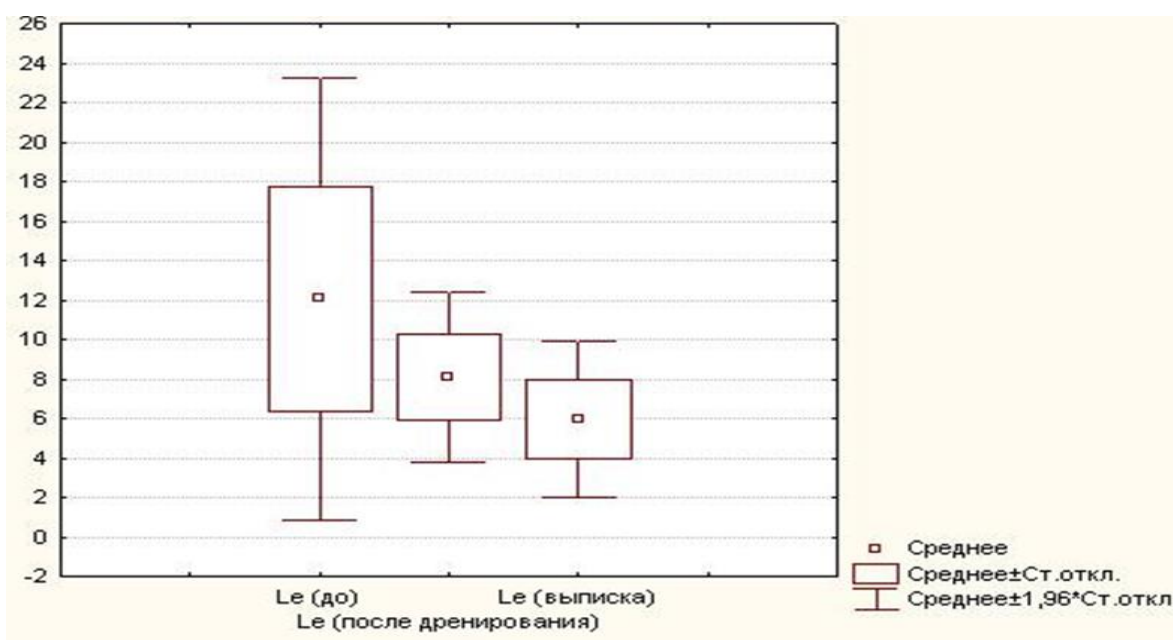

Рис.4. Динамика лейкоцитоза в группе хирургического лечения

Распределение пациентов согласно классификации Hinchey и методам лечения представлено на рисунке 5 .

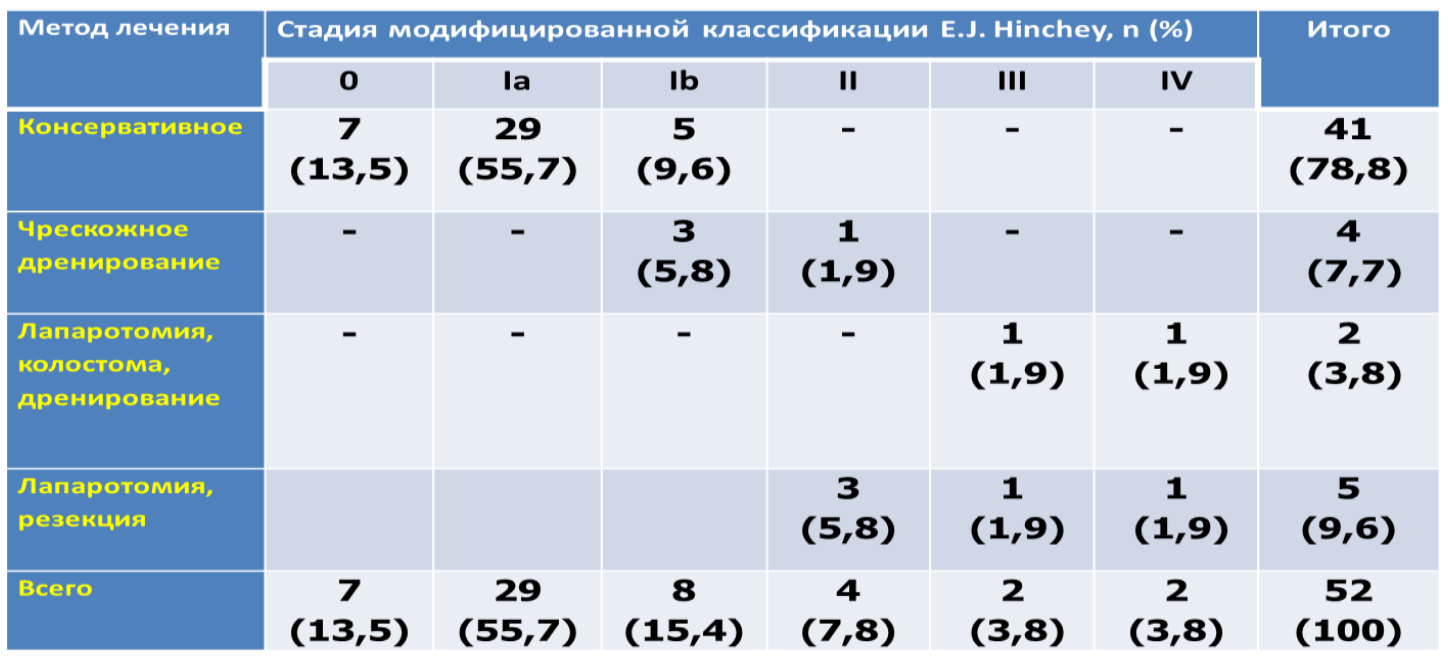

Рис.5. Распределение пациентов согласно классификации Hinchey 
Тактика лечения зависела от размеров абсцесса, выявленного при УЗИ или РКТ. При абсцессах больше 3 см применялись пункционные методы, а абсцессы меньшего размера вели консервативно (рис 6).

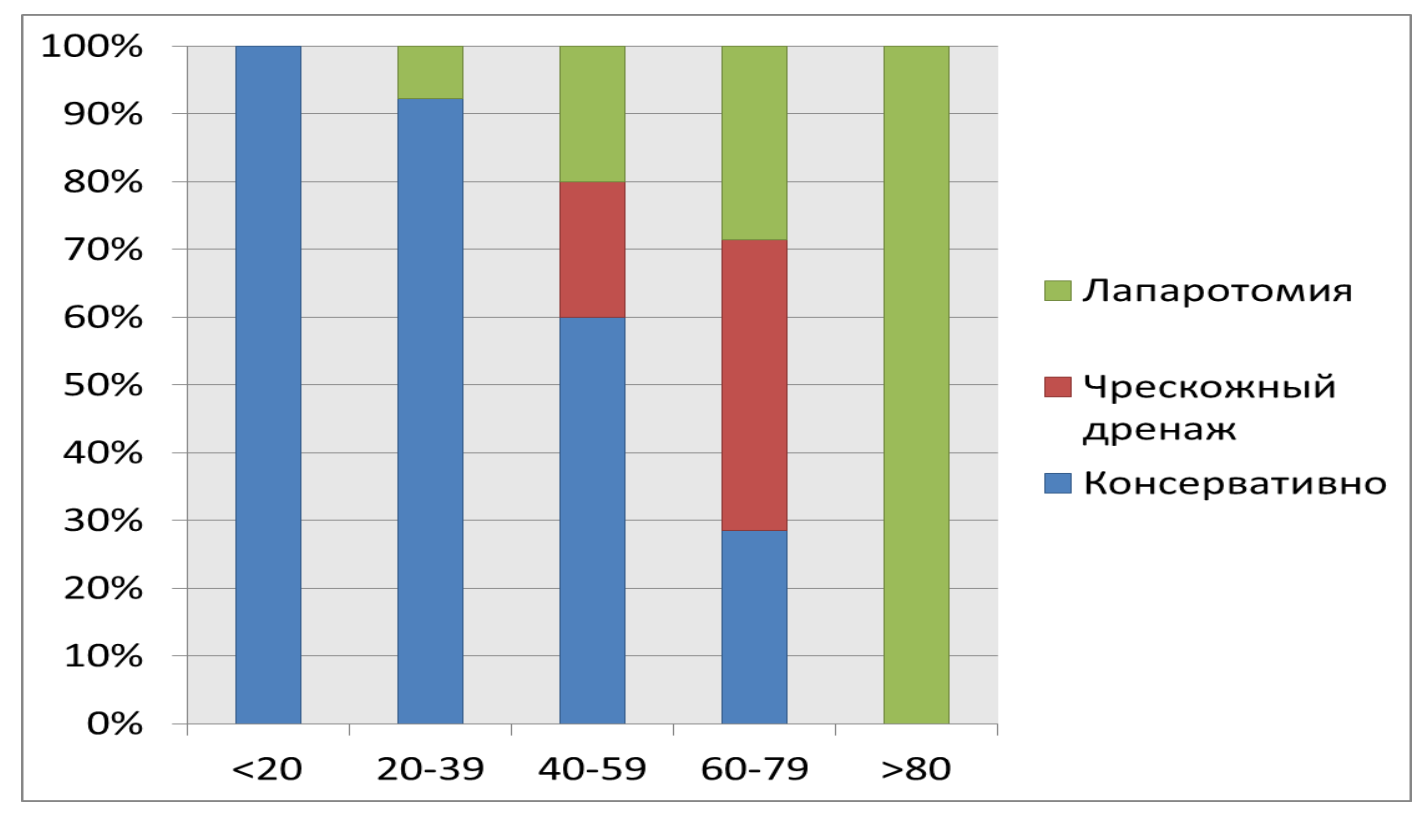

Рис. 6. Зависимость метода лечения от размеров абсцесса

Таким образом, хирургическое лечение показано в случае открытой перфорации кишечника и локального или разлитого перитонита, наличия абсцесса, чрескожное дренирование которого невозможно. При остром дивертикулите и остром паракишечном инфильтрате показано консервативное лечение, целью которого является предотвращение дальнейшего распространения острого воспаления и создание оптимальных условий для эвакуации воспалительного экссудата из дивертикула в просвет кишки. При остром абсцессе до 3 см предпочтение следует отдавать консервативному лечению. При размере абсцесса $\geq 3$ см или отсутствии эффекта от проводимого консервативного лечения показана пункция и дренирование абсцесса под контролем УЗИ или КТ и дальнейшее проведение консервативного лечения.

\section{Список литературы}

1. Tursi A, Papagrigoriadis S. Review article: the current and evolving treatment of colonic diverticular disease. Aliment Pharmacol Ther. 2009 Sep 15;30(6):532-46. doi: 10.1111/j.1365-2036.2009.04072.x. Epub 2009 Jun 22. PMID: 19549266

2. Strate LL, Modi R, Cohen E, Spiegel BM. Diverticular disease as a chronic illness: evolving epidemiologic and clinical insights. Am J Gastroenterol. 
2012 Oct;107(10):1486-93. doi: 10.1038/ajg.2012.194. Epub 2012 Jul 10. PMID: 22777341.

3. Yan Y, Wu JS, Pan S. Age, alcohol, sex, and metabolic factors as risk factors for colonic diverticulosis. World J Clin Cases. 2022 Jan 7;10(1):136-142. doi: 10.12998/wjcc.v10.i1.136. PMID: 35071513; PMCID: PMC8727266

4. Altomare A, Gori M, Cocca S, Carotti S, Francesconi M, Ribolsi M, Emerenziani S, Perrone G, Morini S, Cicala M, Guarino MPL. Impaired Colonic Contractility and Intestinal Permeability in Symptomatic Uncomplicated Diverticular Disease. J Neurogastroenterol Motil. 2021 Apr 30;27(2):292-301. doi: 10.5056/jnm20110. PMID: 33594008; PMCID: PMC8026365..

5. Шелыгин Ю.А., Ачкасов С.И., Москалёв А.И. Классификация дивертикулярной болезни. Колопроктология, №4 (50) 2014, с. 5-13

6. Колопроктология. Клинические рекомендации: клинические рекомендации / Ю.А. Шелыгин [и др.]. - Москва : ГЭОТАР-Медиа, 2015- 528 с

7. Hinchey, E.J., Schaal, P.G. and Richard, G.K. Treatment of perforated diverticular disease of the colon. Advances in Surgery 12:85-109, 1978

8. Krukowski ZH, Matheson NA (1984) Emergency surgery fordiverticular disease complicated by generalized and faecal peri-tonitis: a review. Br J Surg 71:921-927

9. Rezapour M, Stollman N. Antibiotics in Uncomplicated Acute Diverticulitis: To Give or Not to Give?. Inflamm Intest Dis. 2018;3(2):75-79. doi:10.1159/000489631

10. You H, Sweeny A, Cooper ML, Von Papen M, Innes J. The management of diverticulitis: a review of the guidelines. Med J Aust. 2019;211(9):421-427. doi:10.5694/mja2.50276

11. Gregersen R, Mortensen LQ, Burcharth J, Pommergaard HC, Rosenberg J. Treatment of patients with acute colonic diverticulitis complicated by abscess formation: A systematic review. Int J Surg. 2016 Nov;35:201-208. doi: 10.1016/j.ijsu.2016.10.006. Epub 2016 Oct 11

12. Walter Elisei, Antonio Tursi. Recent advances in the treatment of colonic diverticular disease and prevention of acute diverticulitis Ann Gastroenterol. 2016 Jan-Mar; 29(1): 24-32 PMCID: PMC4700842).

13. Marco Ceresoli, Giulia Lo Bianco, Luca Gianotti, Luca Nespoli J Inflamm Res Inflammation management in acute diverticulitis: current perspective. 2018; 11: 239-246. Published online 2018 May 30. doi: 10.2147/JIR.S142990 PMCID: PMC5985778 
14. (Galetin T, Galetin A, Vestweber KH, Rink AD. Systematic review and comparison of national and international guidelines on diverticular disease. Int $\mathbf{J}$ Colorectal Dis. 2018 Mar;33(3):261-272. doi: 10.1007/s00384-017-2960-z. Epub 2018 Jan 18.

15. Cirocchi R, Afshar S, Di Saverio S, et al. A historical review of surgery for peritonitis secondary to acute colonic diverticulitis: from Lockhart-Mummery to evidence-based medicine. World J Emerg Surg. 2017;12:14. Published 2017 Mar 9. doi:10.1186/s13017-017-0120-y

(C) К.Н. Коротких, 2022 